\title{
ATR-mediated regulation of nuclear and cellular plasticity
}

\author{
Gururaj Rao Kidiyoor ${ }^{\mathrm{a}, \mathrm{d}}$, Amit Kumar ${ }^{\mathrm{b}, \mathrm{c}, *}$, Marco Foiani ${ }^{\mathrm{a}, \mathrm{d}, * *}$ \\ a Istituto FIRC di Oncologia Molecolare, Milan, Italy \\ b Developmental Toxicology Laboratory, Systems Toxicology and Health Risk Assessment Group, CSIR-Indian Institute of Toxicology Research, M.G. Marg, \\ Lucknow 226001, India \\ ${ }^{\mathrm{c}}$ Academy of Scientific and Innovative Research (AcSIR), India \\ d University of Milan, Milan, Italy
}

\section{A R T I C L E I N F O}

\section{Article history:}

Available online $\mathrm{xxx}$

\section{Keywords:}

PIKK

ATR

Cellular plasticity

Nuclear envelope

Nucleolus

Centrosome

\begin{abstract}
A B S T R A C T
ATR (Ataxia Telangiectasia and Rad3-related) is a member of the Phosphatidylinositol 3-kinase-related kinases (PIKKs) family, amongst six other vertebrate proteins known so far. ATR is indispensable for cell survival and its essential role is in sensing DNA damage and initiating appropriate repair responses. In this review we highlight emerging and recent observations connecting ATR to alternative roles in controlling the nuclear envelope, nucleolus, centrosome and other organelles in response to both internal and external stress conditions. We propose that ATR functions control cell plasticity by sensing structural deformations of different cellular components, including DNA and initiating appropriate repair responses, most of which are yet to be understood completely.
\end{abstract}

(c) 2016 The Authors. Published by Elsevier B.V. This is an open access article under the CC BY-NC-ND license (http://creativecommons.org/licenses/by-nc-nd/4.0/).

\section{Introduction}

\subsection{ATR, a PI3K like kinase}

ATR is a eukaryotic gene (Chromosome location 3q23), encoding the DNA damage checkpoint protein kinase ATR. ATR consists 2644-amino acids and shares significant homology to previously reported kinases Mei-41 (Drosophila melanogaster), Mec1 (Saccharomyces cerevisiae) and Rad3 (Schizosaccharomyces pombe) [1-3]. The key role of this protein is to maintain genomic integrity. Once activated ATR protects the integrity of replicating chromosomes by stabilizing stalled replication forks and by influencing origin firing and it coordinates cell cycle progression with DNA repair options [4]. ATR is essential for cell survival and its depletion leads to embryonic lethality in mice [5,6]. However, ATR hypomorphic mutations which result in significant reduction of protein levels

Abbreviations: ATR, Ataxia Telangiectasia and Rad3-related; PIKK, Phosphatidylinositol 3-Kinase-related Kinases; PTM, Post Translation Modification; sSDNA, single stranded DNA; ATRIP, ATR interacting protein; RPA, Replication Protein A; DDR, DNA damage response; HEAT, Huntingtin, Elongation factor 3 Alpha-regulatory subunit of protein phosphatase 2A and TOR1; FAT, domainFRAPATM-TRRAP domain; NE, Nuclear Envelope.

* Corresponding author at: Developmental Toxicology Laboratory, Systems Toxicology and Health Risk Assessment Group, CSIR-Indian Institute of Toxicology Research, M.G. Marg, Lucknow 226001, India.

** Corresponding author at: Istituto FIRC di Oncologia Molecolare, Milan, Italy.

E-mail addresses: amit.kumar@iitr.res.in (A. Kumar), marco.foiani@ifom.eu (M. Foiani). cause the Seckel syndrome (OMIM 210600) [7], a rare autosomal recessive disorder, characterized mainly by mild to severe mental retardation, growth retardation, dwarfism, microcephaly and other craniofacial abnormalities $[7,8]$.

ATR belongs to the Phosphatidylinositol 3-kinase-related kinases (PIKKs) family, with a consensus phosphorylation site at serine or threonine residues, that are followed by glutamine (SQ/TQ) [9]. The mammalian PIKK family constitutes of six proteins, namely: mammalian target of rapamycin (mTOR), ataxiatelangiectasia mutated (ATM), ATR, DNA-dependent protein kinase (DNA-PK), suppressor of morphogenesis in genitalia-1 (SMG1 ), and transformation/transcription domain-associated protein (TRRAP). Unlike canonical PI3 kinases, the PIKKs lack lipid phosphorylation activity and function as protein kinases instead [10].

During the past two decades, studies on the physiological role of ATR were mainly focused on its role as a DNA damage response kinase in the context of genotoxic insults, although more recently it has been implicated in other processes [11,12]. We will summarize the mechanisms leading to ATR activation and discuss the noncanonical modes of ATR activation in response to various intrinsic and extrinsic cellular stresses.

\subsection{Canonical activation of ATR}

In the last couple of decades, the roles of ATR in maintaining genome integrity by ensuring replication fork stability and coordinating cell cycle progression with the DNA repair has been extensively studied [4,13-15]. Important mechanistic details on ATR functions have been elucidated in budding and fission yeasts 

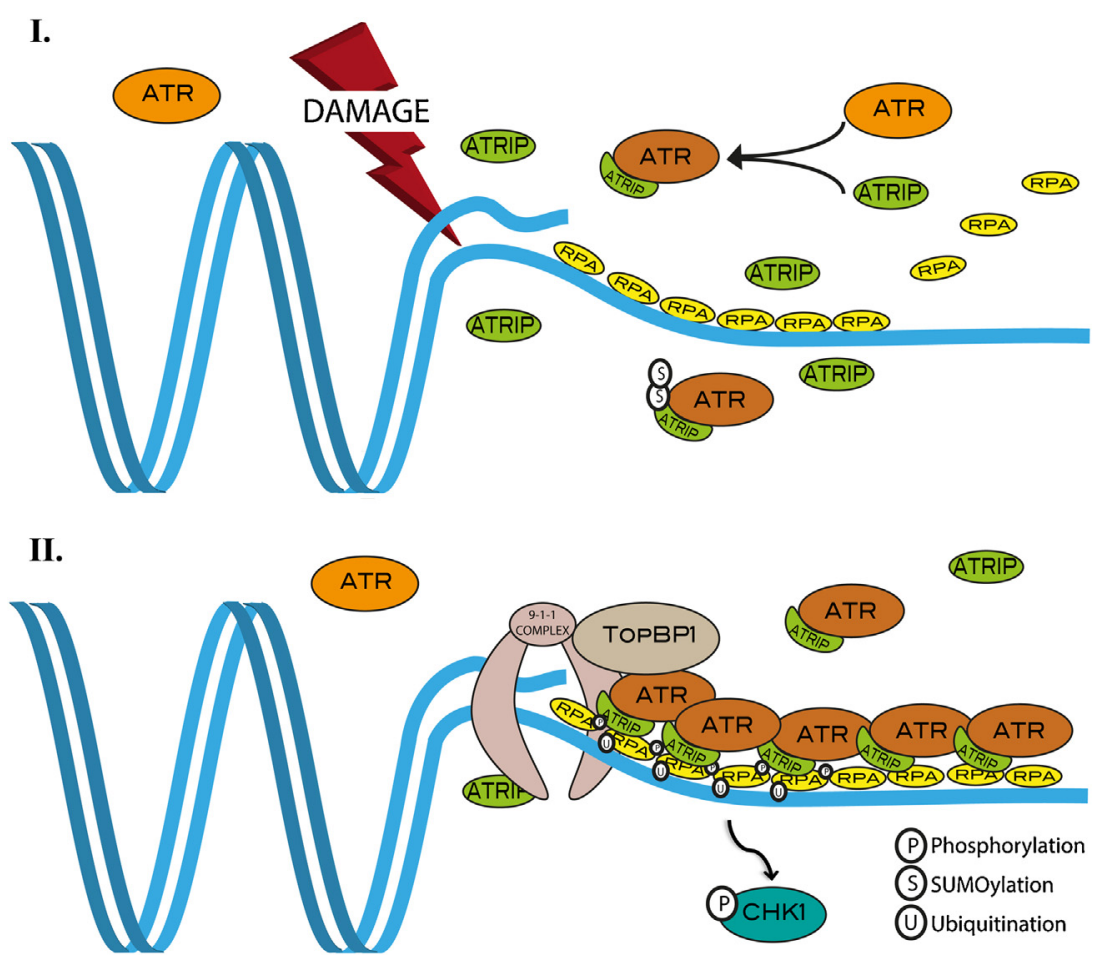

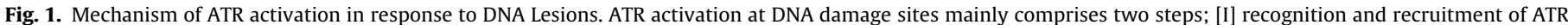

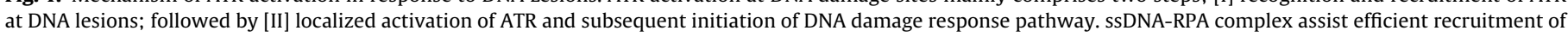

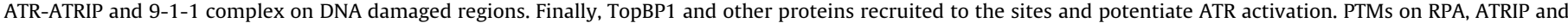
ATR (depicted) also play a crucial role (feed back loop) in achieving complete ATR activation.

where it is called Mec1 and Rad3 respectively. Along with ATM and DNA-PK, ATR is activated in response to DNA damage and initiates a cell response leading to cell cycle slowdown, stimulation of DNA repair and induction of apoptosis in the presence of massive DNA damage. ATR specifically recognises single stranded DNA (ssDNA) lesions coated by RPA (Replication Protein A). Processes like DNA replication and DNA damage repair commonly give rise to such ssDNA (for detailed reviews refer to $[4,13,14]$ ). However, whether ssDNA represents the ATR signal is still under debate [16-18]. ATR activation in response to DNA damage is dependent on its binding with the obligatory partner ATRIP (ATR interacting protein) [19]. Briefly (Fig. 1), genotoxic agents such as DNA damage inducers or drugs interfering with DNA synthesis as well as pathological events such as oncogene-induced replication stress, generate long stretches of ssDNA that becomes immediately coated with the ssDNA binding protein complex RPA. RPA-ssDNA nucleofialments act as a substrate for ATR, which is recruited there along with its partner ATRIP $[20,21]$ in a 9-1-1 complex and TOPBP1-dependent manner [4,22] (Fig. 1). Once recruited, ATR phosphorylates an array of proteins including the checkpoint kinase 1(CHK1) [23] thus triggering the cellular level response to DNA damage.

While activation of ATR following DNA damage is primarily assisted by its heterodimerization with its obligatory partner ATRIP, and subsequent associations with RPA-ssDNA nucleofilaments and with TopBP1 $[19,20]$, recent observations suggest that, perhaps, this scenario is rather oversimplified. ATR activation is influenced also by post translational modifications (PTM) such as ubiquitination of RPA [24] and SUMOylation of ATRIP [25]. Inhibition of RPA32 ubiquitination through depletion of the ubiquitin ligase PRP19 (Pre-mRNA-processing factor) diminishes RPA complex phosphorylation and results in limited recruitment and activation of the ATR complex on RPA-ssDNA [24]. Moreover, ATRIP SUMOylation mutants reduce ATRIP interactions with ATR as well as with RPA and TopBP1. Some studies also support the notion of an alternative pathway, independent of RPA, for ATR activation in response to DNA damage $[17,18,26]$, suggesting that further studies are needed to understand the DNA damage-dependent role of ATR and to revisit the currently accepted model in the field.

\subsection{Non-canonical activation of ATR}

Since PI3K members are sensors of distinct cell stress responses, PIKKs, including ATM, ATR and DNA-PKs are also expected to perform multiple functions, rather than being confined to a particular one. However, due to the elevated attention towards the DNA damage responses (DDR), possible alternative roles for these kinases have been perhaps overlooked. These kinases are also expressed in terminally differentiated tissues such as muscles and neurons, which do not undergo any active cell division. The symptoms of gene-associated diseases such as ataxia telangiectasia progressively increase after the complete development, arguing for a DNA damage-independent role of these kinases [27,28]. Moreover, few studies confirmed the presence of ATM, ATR and DNA-PK in the cytoplasm and in organelles, contributing in their respective functionalities in DNA damage-independent pathways [27,29-31]. ATR has been shown to respond to a variety of stress stimuli like mechanical stress, osmotic stress [12] and thermal shock [32] (Figs. 3 and 4). In addition, ATR was shown to play important roles in organelles like mitochondria [11], centrosomes [29] and nucleoli $[12,33]$.

Below, we summarize all the alternative substrates that are proposed to activate ATR other than DNA.

\section{Structure and PTMs of ATR}

PIKKs share a conserved primary structure with a varying number of amino acids in each domain (Fig. 2). The general structure of PIKKs comprises of a N-terminal HEAT (Huntingtin, Elongation 
ATR

\begin{tabular}{|c|c|c|c|c|c|c|c|}
\hline \multicolumn{3}{|c|}{ Amino Acids } & Approx. 1- 1500 & $1640-2185$ & $2322-2567$ & $2568-2597$ & $2612-2644$ \\
\hline $\begin{array}{c}\text { ATRIP } \\
\text { Binding }\end{array}$ & NLS & $\begin{array}{l}\text { BH3 } \\
\text { like }\end{array}$ & HEAT repeats ( $\alpha$-Solenoid) & T & SE & & \\
\hline
\end{tabular}

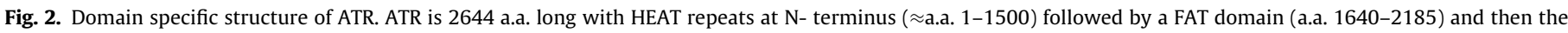

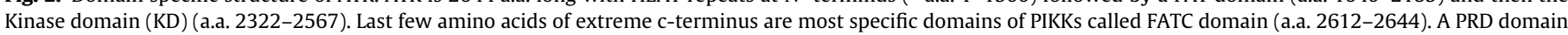
(a.a 2568-2597) that regulates the activity of the protein is located in-between the Kinase domain and the FATC domain.

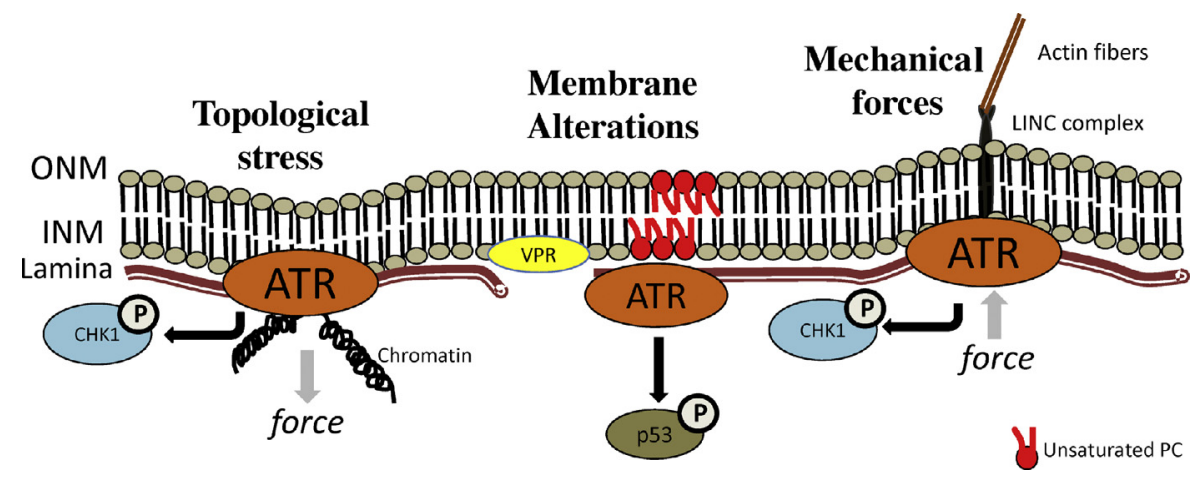

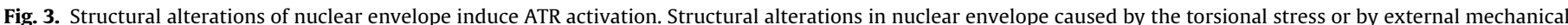

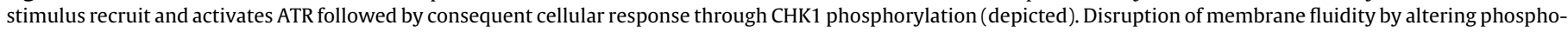

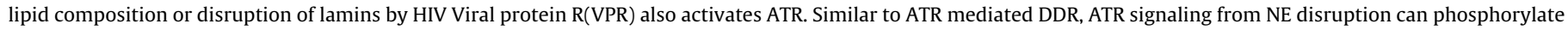
proteins like CHK1 and p53 and induce cellular response such cell cycle arrest.

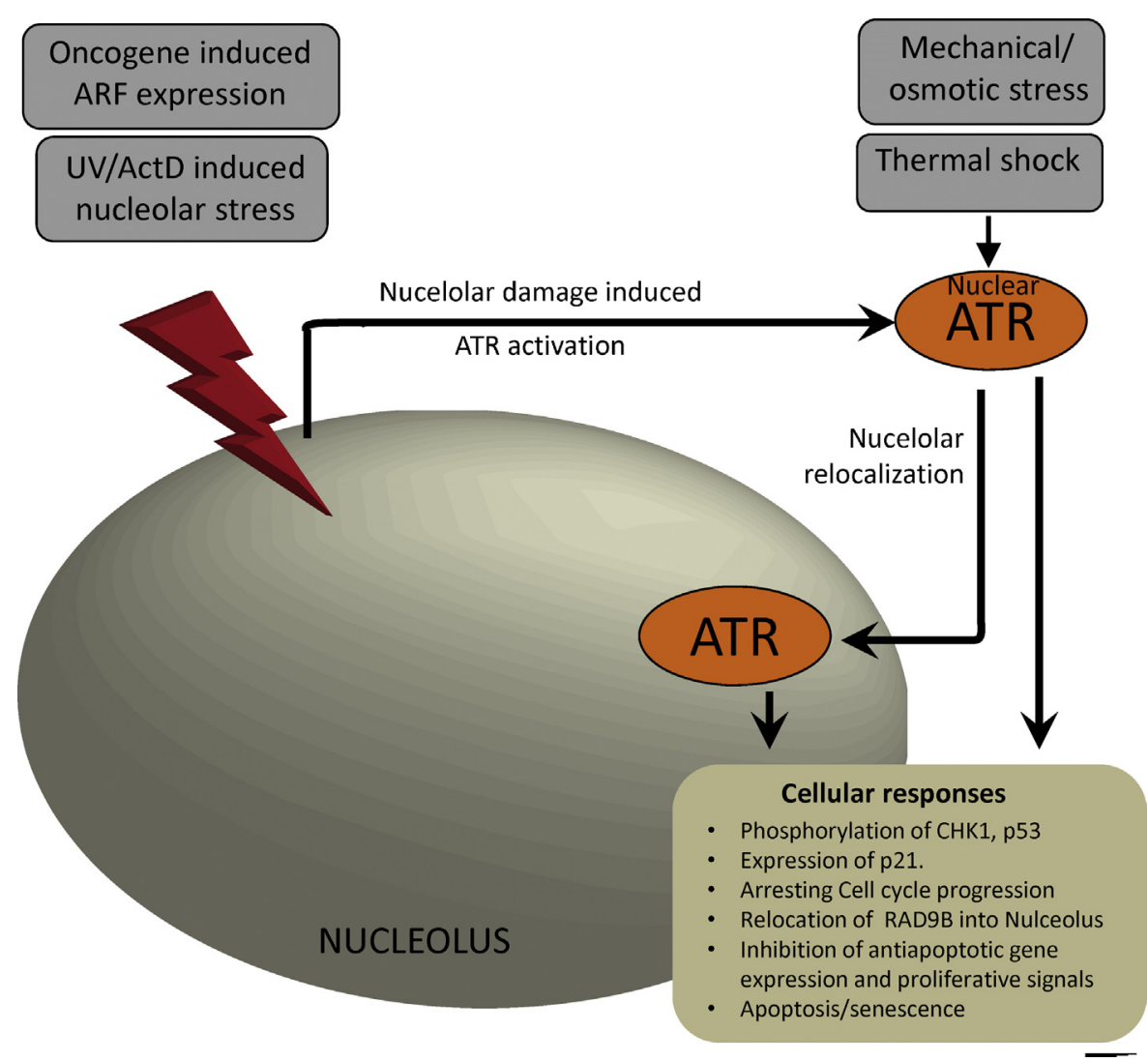

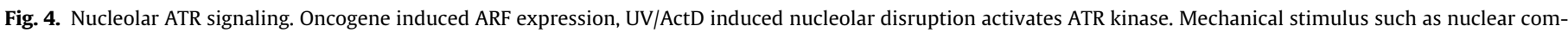

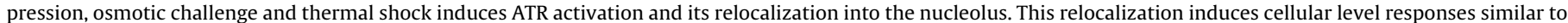
DDR and can help in recruitment of other proteins into the nucleolus. 
factor 3, Alpha-regulatory subunit of protein phosphatase $2 \mathrm{~A}$ and TOR1) repeats followed by FAT domain (FRAP-ATM-TRRAP), KD (kinase domain), PRD (PIKK regulatory domain) and a FATC (FATC-terminal) domain [4,34-36] (Fig. 2). In general, HEAT repeats mediate protein-protein interactions and the large number of HEAT repeats in PIKKs might indicate the presence of a large set of interactive substrates [36-38]. In the case of ATR, its N-terminus constitutes of 45 HEAT repeats [37], including the binding site for ATRIP and a Nuclear Localization Sequence (NLS) (Fig. 2) [4]. ATRIP binding to ATR is dispensable for the activity of ATR [4,19]. The ATRIP-ATR complex also provides a platform for post translational modifications such as phosphorylation, SUMOylation [25] and ubiquitylation [24] that enhance the binding of other interactors such as RPA and TopBP1. A recent study reported the presence of a BH-3-like domain within HEAT repeats, which may explain the role of ATR in regulating apoptotic processes [11]. The HEAT repeats of DNA-PK have been proposed to bind DNA $[39,40]$. Despite the fact that ATR does not contain any DNA-binding domains, it can interact with UV-damaged DNA [41]. Extrapolations from the structure of DNA-PK can propose a potential region in ATR for DNA binding [18]. A point mutation within HEAT repeats (S1333) resulted in a hyperactive ATR kinase suggesting that HEAT repeats can alter the kinase activity of ATR [42]. Intriguingly, HEAT repeats also acts as elastic connectors, ideal to sense mechanical stimuli [43]. The FAT domains of mTOR and DNA-PK are composed mainly of Helix-turnHelix repeats and they wrap around the kinase domain [38-40]. This could apply to other PIKKs including ATR [38]. Both ATM and ATR interact with NEMO (NF-kB essential modulator) through the FAT domain and regulate NF-kB activity [44], suggesting that the FAT domain can regulate binding partners in certain contexts. The kinase domain (KD) of PIKKs shares maximum homology with the catalytic domain of classical PI3 Kinases. In spite of this homology, kinases of the PIKK family lack lipid kinase activity; rather they catalyze protein phosphorylation upon activation [34,35]. Every PIKK contains a unique amino acid sequence between the Kinase domain and the FATC domain defined as the PIKK Regulatory domain (PRD) $[34,35]$. The PRD domain of ATR is hypothesized to be 30 amino acids long and to interact with TopBP1 [45]. Mutations in the PRD of ATR affect the TopBP1-dependent ATR activation without influencing its basal kinase activity and ATRIP binding [45]. The most C-terminal of ATR has a FATC domain, the most conserved domain amongst all the PIKK members (Fig. 2). This 33-amino acid long domain, consisting mostly of hydrophobic aromatic residues, is dispensable for PIKK kinase activity [34,35]. Resembling PI3 kinases, the FATC domains of all PIKKs can interact with membrane mimetic structures and induce structural alterations within them [46]. FATC domains are not only conserved but functionally identical amongst PIKK members and can be interchanged without affecting the kinase activity, at least for some PIKKs [47]. However, replacing the FATC domain of ATR with that of ATM results in a kinase-dead protein, suggesting that the FATC domain of ATR is both unique and necessary for its activity [45]. In yeast, the FATC domain of Mec1 mediates its kinase activity, nuclear localization and stability of the protein [48].

ATR, given its large size harbors many potential targets for PTM. Despite the fact that these PTMs could alter the function, conformation and structure of ATR, our knowledge regarding the PTMs and their relevance remains very limited. Similar to other PIKKs, ATR also undergoes autophosphorylation at Thr-1989 upon DNA damage, a proposed hallmark for active ATR [49,50]. This autophosphorylation is strongly dependent on the kinase activity of ATR and could impact the ATR-dependent DNA damage response. Another phosphorylation site is Ser-428, which is implicated in mRNA processing [51] and in altering ATR isomerization [11]. These recent findings mark the beginning of identifications of different PTMs that regulate ATR activity; an expanding area of research. Insights from the structure and the PTM status of ATR will have a profound impact on our understanding of its activity.

\section{NON-canonical substrates for ATR activation}

\subsection{Nuclear envelope}

The nuclear envelope (NE) is a phospholipid bilayer that separates the nucleoplasm from the cytoplasm. It is composed of a bilayer embedded with nuclear pores, Lamins and LINC complexes (for detailed review Refs. [52,53]). Apart from its role in regulating nuclear homeostasis, the NE also functions as a signal inducer, integrator and transducer of a variety of cellar signaling pathways [54]. It influences nuclear transport, transcription, replication, DNA repair, protein export, mRNA synthesis, cell fate/differentiation and migration among other processes. The structure and composition of NE are strictly regulated based on the tissue and the cell cycle phase [54-58]. Events that challenge NE integrity could lead to a variety of pathologies including cancer [53-55,59].

The NE constantly experiences mechanical forces originating internally by topological constrains of chromatin or by external stimuli on the cytoskeleton [53]. LINC complexes in the NE connect the nucleus with the cytoskeleton providing continuity for transducing mechanical signals across the membranes [60]. This makes the NE an ideal platform to sense both nuclear and cytoskeletal mechanical stress and, if needed, to convert them into a chemical signal, which can then be exploited by the cell for initiating an appropriate response.

During mitosis, condensing chromatin impose topological stress to the NE [61]. Through an unknown mechanism, ATR can sense these forces, relocate into the NE and locally phosphorylate CHK1 kinase at the NE [12] (Fig. 3). In S. cerevisiae, Mec1 resolves fork collision with transcription machinery near the NE by untethering the RNA from the nuclear pore and relaxing the DNA [62]. Furthermore, ATR also responds rapidly to NE deformation caused by external forces [12] or altered membrane fluidity [63] (Fig. 3). Hyperosmotic conditions shrink the nucleus and induce raffles in the NE. Under this hyperosmotic stress conditions, ATR relocates into the NE and phosphorylates CHK1 locally [12] in a reversible way. This osmotic challenge is also known to induce chromatin compaction [64], suggesting an involvement of ATR in this process as a measure to preserve genome integrity [12]. Phosphorylated ATR (S428) is present in the nucleus with little enrichment in the NE and in splicing speckles [51]. However, compromising the integrity of NE by deleting Lamin A results in a strong reduction of this phosphoATR signal, suggesting the NE can alter the PTM status of ATR and influence its function [51]. Another study demonstrates that ATR is activated upon altering lipid composition of membranes [63] (Fig. 3). Phosphatidylcholines (PCs), a class of phospholipids composing membranes including nuclear envelope, constitute one of the most abundant phospholipid bulks in membranes [65]. The nuclear membrane has a high proportion of saturated PCs relative to polyunsaturated PCs. Altering membrane fluidity, by reducing levels of saturated PCs and increasing polyunsaturated PCs using a specific inhibitor leads to ATR activation, p-53 phosphorylation and cell cycle arrest in G1 phase [63]. Transfecting cells with HIV-1 Viral protein R (VPR) also resulted in ATR activation and subsequent $\mathrm{CHK} 1$ phosphorylation and cell cycle arrest during $\mathrm{G} 2$ of the cell cycle [66] (Fig. 3). Localization of VPR to the NE, which results in NE disruption, is crucial for this arrest $[67,68]$. This suggests that ATR can directly or indirectly sense NE deformations [69].

Hence, both physical and chemical alterations of the nuclear envelope can activate ATR (Fig. 3) and subsequent signaling such as phosphorylation of $\mathrm{CHK} 1, \mathrm{p} 53$ and cell cycle control. In addition, there is an overlap of cellular processes where both ATR and NE 
are involved. Even though most of the above-mentioned studies are centered on the classical role of ATR such as phosphorylation of CHK1 and p53 and cell cycle arrest, they strongly emphasize the need for further characterization of ATR's role in NE homeostasis. Several questions like the mechanism of ATR recruitment and activation at the altered $\mathrm{NE}$, proteins and pathways that are recruited by active ATR at the NE and most importantly the physiological relevance of ATR's role at the NE in a living organism remains to be addressed.

\subsection{The nucleolus}

Nucleoli are non-membranous organelles in the nucleus harboring mainly ribosomal genes (rDNA). Nucleolar homeostasis is determined both by its apt functionality and well-preserved morphology. Apart from its main role in ribosomal biogenesis, nucleolus also functions as a sensor of stress for the cell. Almost all types of cellular stress (as comprehensively listed by Boulon et al. [70]) alter nucleolar morphology or function, threatening its homeostasis. This in turn triggers response mechanisms within and outside the nucleolus. These responses include inhibition of rRNA synthesis, inhibition of cell cycle progression, structural alterations, disassembly and reorganization of compartments, segregation and protein trafficking [70]. PIKKs can also mediate some of these responses $[71,72]$. Inhibition of mTOR by nutrient deprivation or rapamycin treatment reduces ribosome subunit production [72]. ATM is a central player for repairing nucleolar DNA lesions and in regulating rRNA synthesis in response to non-nucleolar DNA damage [71]. ATR instead functions in stress stimuli that are independent of DNA damage, responding mainly to alterations in nucleolar homeostasis (discussed below).

ATR responds to nucleolar stress either by re-localizing itself into the nucleolus or by phosphorylating CHK1 and p53, stabilizing p53 and consequently slowing down cell cycle progression. Recent studies showed that ATR relocates into the nucleolus in response to external stress conditions that alter nucleolar homeostasis (Fig. 4). When cells are challenged with hypoosmotic stress or mechanical stress by applying compressive load, ATR rapidly relocalizes into the nucleolus as well as at the nuclear envelope and phosphorylates CHK1 [12]. In addition, thermal shock (hypothermia) activates ATR, which relocates into the nucleolus, phosphorylates p53(S15), consequently up-regulating p21 expression and inducing cell cycle arrest [32]. Inhibition of RNA polymerase I with actinomycin $\mathrm{D}(\mathrm{ActD})$ also induces nucleolar stress. This leads to activation of ATR, which then phosphorylates CHK1(S345) and p53(S15) [33,74]. Moreover, Rad9B (a Rad9A paralog), relocates on nucleoli in a ATR and JNK dependent manner upon UV-induced nucleolar disruption [75], suggesting that ATR can also recruit other proteins into damaged nucleoli. Ectopic expression of TopBP1 [74] induces nucleolar stress by inhibiting rRNA synthesis and causes nucleolar segregation through a process dependent on ATR and possibly the 9-1-1 complex.

ARF tumor suppressor ( $14^{\mathrm{ARF}}$ ) is a nucleolar protein that inhibits rRNA synthesis, induces anti-proliferative geneexpression, activates pro-apoptotic signals and regulates replicative and oncogene-induced senescence[76-78]. Upon induction, ARF activates the ATR/CHK1 pathway (Fig. 4), which then deactivates RelA, (a component of NF-kB), by phosphorylating it (T505). ARF induction also results in nucleolar relocalization of ATR along with BRCA1, and in phosphorylation of p53(S15) and cell cycle arrest [79]. In a similar study on cells lacking the MAPK members ERK1 or ERK2, ATR re-localized in the nucleolus instead of ssDNA-RPA foci upon HU treatment [80]. The MEK-ERK pathway is activated by many growth factor signals and is shown to regulate the DDR activity of ATR [81] through an unknown mechanism. Deletion of ERK1 and 2 reduces replication-induced CHK1 and p53 phosphorylation without affecting p53 stabilization. Another study demonstrated that in senescent cells, ATR is localized to the nucleolus, and this subpopulation of nucleolar ATR does not exhibit phosphorylation at Ser-428 [51].

In summary (Fig. 4), ATR senses nucleolar integrity. Since the only parameter that is common in all the above cases is an alteration of structural integrity of the nucleolus, without any obvious DNA damage (observed by the $\mathrm{yH} 2 \mathrm{AX}$ foci or 53BP1 foci formations), one possibility is that ATR senses such structural alterations triggering an appropriate response. However, more work will be required to fully elucidate the ATR-mediated response to nucleolar stress.

\subsection{Centrosome}

Centrosomes are the microtubule organizing centers in the cytoplasm, composed of two centrioles coated with a matrix of pericentriolar material (PCM). Other than regulating microtubule nucleation and mitotic spindle formation, centrosomes also function as decision makers in many cellular processes [82,83]. The number of centrosome and their duplication are tightly regulated in a cell cycle-dependent way. Altered numbers of centrosomes (as in case of many cancers) could result in multipolar cell divisions, aneuploidy and mitotic catastrophe. Centrosome duplication, DNA damage and cell cycle seem to have a very intricate dependency. Several DNA damage signaling molecules, cell cycle regulators and checkpoint kinases are localized to centrosomes and/or functionally regulated by centrosomes [84]. Many carcinogens and DNA damaging agents alter this centrosome number and/or alter the centrosome duplication cycle [84]. (However, we will focus only on ATR and its DDR-independent functions, for detailed review on centrosomes please read [82-84]).

During mitosis, ATR and other members of this pathway ATRIP, CHK1, p53 and BRCA1 localize to the centrosomes [29]. Centrosomal ATR was phosphorylated at Ser-428 [51]. Furthermore, NBS1/ATR/BRCA1 pathway is necessary for mono-ubiquitination of $\gamma$-Tubulin and for regulating centrosome amplification [85]. Immunoprecipitation of ATR with $\gamma$-Tubulin suggests a direct role in regulating tubulin-nucleation process [29]. Cells from ATR seckel patients have supernumerary centrosomes and defective mitosis [86]. Seckel syndrome is also caused by mutations affecting the gene encoding the centrosomal protein Pericentrin (PCNT), which is involved in the ATR-dependent signaling pathway [87]. Surprisingly, PCNT-seckel patients also show defective ATR signaling pathway $[86,88]$. Other centrosomal gene mutations including CEP152, CPAP, MCPH1 also cause microcephaly and dwarfism. Most of them, if not all, are involved in ATR signaling and patient cells have defective ATR signaling [89].

Centrosomes play a prominent role in deciding symmetric and asymmetric cell divisions and cell fate by regulating spindle alignment [82]. In Caenorhabditis elegans, ATR/CHK1 pathway regulates asynchrony in cell division by differentially inducing replication delays in cells [90]. Forced expression of ATR inhibits MyoD, consequently counteracting differentiation of myoblasts into muscles. Additional phenotypes include abnormal centrosomal amplifications, aneuploidy and defective cell cycle arrest [91]. However, the contributions of centrosomes in these contexts seem highly plausible, but remain to be tested. In non-dividing, quiescent cells, centrioles form basal bodies for cilia and flagella [82,83]. A recent study reported ATR localization in the cilia of Photo Receptor (PR) cells of mice retina. They further showed that the absence of ATR localization to cilia in heterozygous ATR seckel mice resulted in significant shortening of cilia and as a result PR cells underwent severe degeneration [31], suggesting a possible role of ATR in maintaining cilia in non-dividing cells.

The relation between ATR and centrosomes seem to be more complicated than other structures discussed earlier, the NE and 
the nucleolus. Both ATR and centrosomes can influence each other functions significantly, thus impacting at the organismal level. Hence, studies uncovering the crosstalk of ATR and centrosomes are necessary and clinically relevant.

\subsection{The cytoplasm}

A recent study showed that cytoplasmic ATR is associated with mitochondria and through $\mathrm{BH} 3$ domain-dependent interactions with mitochondrial proteins, it promotes anti-apoptotic pathways [11]. In C. elegans ATL-1(ATR ortholog) defects cause reduction of mitochondrial DNA copy number and induce activation of mild oxidative stress and altered lifespan of the organism $[92,93]$. Through its FAT domain, ATR can interact with NEMO and suppress the pro apoptotic signaling in the context of replication stress [44]. In brain tissues, ATR was shown to be more cytoplasmic, interacting with ATM and phosphorylating the synaptic vesicle protein VAMP2, suggesting a role in neuronal synapses [30].

ATR seckel fibroblasts have active p38 stress response and as a result undergo premature senescence. These cells also express elevated levels of stress fibers, increased levels of phospho-Cofilin, a regulator of actin dynamics and endocytotic protein phoshoCaveolin1 [94]. In D. melanogaster, Mei-41 was identified in a screen as one of the cytoskeletal organization factors [95]. In addition, a large number of cytoskeletal proteins are phosphorylated by ATM/ATR upon genomic stress [23]. Recently a study revealed a crosstalk between ATR and Rho signaling. Alterations in the Rho pathway resulted in reduced ATR signaling and ATR defects reduced active RhoA and phosho-myosin light chain 2, implying defective Rho signaling [96]. Another novel role of ATR is in regulating leptindependent STAT3 signaling, suggesting a potential involvement in maintenance of cellular energy balance [97].

The number of cellular processes involving or regulated by ATR are constantly on the rise. However, the majority of them are based on preliminary pieces of evidences and further studies need to be performed in order to have a clear unified understanding of the role of ATR in the cytoplasm.

\section{Implications in diseases and clinical relevance}

Since ATR is an essential gene, it is rare to find a loss of functionassociated diseases with this gene. Seckel syndrome is one such rare disorder that is associated with ATR and is caused by a splicing mutation (Exon 9) [7] or heterozygous mutations combining deletion in one allele and splicing mutation in another allele (exon 33) [98]. As a result, seckel cells exhibit a significant reduction in the level of ATR. Seckel patients suffer severe growth retardation, microcephaly and defective brain and central nervous system development [8]. Other mutations that cause microcephaly and dwarfism alter centrosome proteins like CENPJ, PCNT and CEP152. However, it is important to note that the majority of them have defective ATR signaling. It was also proposed that the pathway involving ATR, centrosome and cell cycle progression could be the key central theme for causing primary dwarfism [89]. Additionally, blepharophimosis-ptosis-epicanthus inversus syndrome (BPES), another syndrome with microcephaly and growth retardation, caused by deletions of FOXL2 gene at chromosomal locus 3q, some of the non-BPES abnormalities were assigned to the deletion of ATR, which is also at the same chromosomal locus 3q [99]. This raises the possibility of secondary level mutations in ATR in diseases that are not yet related to ATR. Mouse models with conditional deletion of ATR or humanized Seckel mice show premature aging or progeroid symptoms $[100,101]$. Generally, defective NE is considered to be the cause of these syndromes, and patient cells exhibit compromised nuclear architecture, defective DDR pathways, replication arrests and senescence [102]. Since ATR can directly sense the NE deformations and induce responses and alter its localization and function in normal and senescent cells, understanding the role of ATR in the context of these laminopathies will be relevant.

Traditionally, ATR is considered a tumor suppressor. However deletion of ATR can reduce tumor development and progression [103]. Inhibitors of ATR kinase activity are actively perceived as anti-cancer drugs [104]. But, certain ATR mutations that do not alter protein levels can increase the probability of cancer disposition, [105]. Cancer databases also shed light on the large array of point mutations in the ATR gene that occur in variety of tumors, and do not necessarily alter the level of the protein. These mutations can have a significant impact on the function of ATR. Sarcoma cells phenotypically fail to terminally differentiate and forced expression of ATR in myoblasts mimics this scenario by inhibiting differentiation through inhibiting MyoD [91]. Altogether, based on these observations, we can conclude that depending on the level and integrity of protein, ATR can function both as tumor suppressor or oncogene. However, insights in this particular direction will have a larger impact in therapeutics and targeted drug design.

\section{Conclusions and future prospective}

Although ATR is considered to be a DNA damage response kinase, it is clear that it plays a relevant role in a variety of cellular aspects independent of DNA damage. However, at present, we have less understanding of the molecular basis underlying such non-canonical ATR pathways. Most studies focus on the role of ATR in cell cycle arrest and phosphorylation of p53 and CHK1, but there is a need to understand further how ATR is activated in response to different stress stimuli and which are the key targets that are then phosphorylated. Moreover, what are the influences of MAP kinase and other signaling pathways on ATR functions.

Cilia form a major component of mechano and chemo sensing organs of the cell and neurons are one of the largest utilizers of chemical signaling. Presence of ATR in cilia and in neuronal cytoplasm plus spontaneous responses of ATR to mechanical stimuli all suggests a potential involvement of ATR in chemo and mechano signaling process, which needs to be further explored. Intricate crosstalk between ATR and organelles that regulate active cell dynamics like nuclear envelope, centrosomes and cytoskeleton leads to postulate the involvement of ATR in cellular processes such as migration and differentiation. This is also supported by the fact that seckel patients have defective neuronal migration, agenesis, and cardiomyopathy. In addition, overexpression of ATR inhibits myoblast differentiation and the mouse depleted for ATR suffers from stem cell loss. Further insights in this direction will be very useful and also clinically relevant.

To conclude, we propose that the phenotypes and symptoms exhibited by seckel patients and ATR deletion mouse model are a final outcome of accumulated defects due to failures in various cellular processes throughout development and post development, rather than caused by the damaged DNA alone. We hypothesize that ATR has a more general role in controlling cell plasticity and integrity and not only genome integrity. We propose that ATR can sense structural alterations in key cellular components (one of which is DNA) and respond by activating yet to be discovered pathways.

\section{Conflict of interests}

The authors declare no conflict of interest.

\section{Acknowledgements}

We thank G. Shubassi for critical reading of the manuscript and members of MF group the discussions and comments. G.R.K 
is supported by Marie Curie Initial Training Networks (ITN) (FP7 'aDDRess') fellowship. M.F. is supported by grants from AIRC, Telethon-Italy, and the European Commission. Work in A.K. lab is supported by the CSIR-Indepth and -Epigenetics in health and disease network projects and by Department of Science and Technology, India.

\section{References}

[1] K.A. Cimprich, et al., cDNA cloning and gene mapping of a candidate human cell cycle checkpoint protein, Proc. Natl. Acad. Sci. U. S. A. 93 (7) (1996) 2850-2855.

[2] M.F. Hoekstra, Responses to DNA damage and regulation of cell cycle checkpoints by the ATM protein kinase family, Curr. Opin. Genet. Dev. 7 (2) (1997) 170-175.

[3] N.J. Bentley, et al., The Schizosaccharomyces pombe rad3 checkpoint gene, EMBO J. 15 (23) (1996) 6641-6651.

[4] K.A. Cimprich, D. Cortez, ATR: an essential regulator of genome integrity, Nat. Rev. Mol. Cell Biol. 9 (8) (2008) 616-627.

[5] E.J. Brown, D. Baltimore, ATR disruption leads to chromosomal fragmentation and early embryonic lethality, Genes Dev. 14 (4) (2000) 397-402.

[6] A. de Klein, et al., Targeted disruption of the cell-cycle checkpoint gene ATR leads to early embryonic lethality in mice, Curr. Biol. 10 (8) (2000) 479-482.

[7] M. O'Driscoll, et al., A splicing mutation affecting expression of ataxia-telangiectasia and Rad3-related protein (ATR) results in Seckel syndrome, Nat. Genet. 33 (4) (2003) 497-501.

[8] A. Shanske, et al., Central nervous system anomalies in Seckel syndrome: report of a new family and review of the literature, Am. J. Med. Genet. 70 (2) (1997) 155-158.

[9] S.T. Kim, et al., Substrate specificities and identification of putative substrates of ATM kinase family members, J. Biol. Chem. 274 (53) (1999) 37538-37543.

[10] M. De Cicco, M.S. Rahim, S.A. Dames, Regulation of the target of rapamycin and other phosphatidylinositol 3-kinase-related kinases by membrane targeting, Membranes (Basel) 5 (4) (2015) 553-575.

[11] B.A. Hilton, et al., ATR plays a direct antiapoptotic role at mitochondria: which is regulated by prolyl isomerase pin1, Mol. Cell 60 (1) (2015) 35-46.

[12] A. Kumar, et al., ATR mediates a checkpoint at the nuclear envelope in response to mechanical stress, Cell 158 (3) (2014) 633-646.

[13] P. Awasthi, M. Foiani, A. Kumar, ATM and ATR signaling at a glance, J. Cell Sci. 128 (23) (2015) 4255-4262.

[14] D. Branzei, M. Foiani, Maintaining genome stability at the replication fork, Nat. Rev. Mol. Cell Biol. 11 (3) (2010) 208-219.

[15] M.K. Zeman, K.A. Cimprich, Causes and consequences of replication stress, Nat. Cell Biol. 16 (1) (2014) 2-9.

[16] K.M. Sleeth, et al., RPA mediates recombination repair during replication stress and is displaced from DNA by checkpoint signalling in human cells, J. Mol. Biol. 373 (1) (2007) 38-47.

[17] G.E. Dodson, Y. Shi, R.S. Tibbetts, DNA replication defects: spontaneous DNA damage, and ATM-dependent checkpoint activation in replication protein A-deficient cells, J. Biol. Chem. 279 (32) (2004) 34010-34014.

[18] E.A. Nam, D. Cortez, ATR signalling: more than meeting at the fork, Biochem. J. 436 (3) (2011) 527-536.

[19] D. Cortez, et al., ATR and ATRIP: partners in checkpoint signaling, Science 294 (5547) (2001) 1713-1716

[20] L. Zou, S.J. Elledge, Sensing DNA damage through ATRIP recognition of RPA-ssDNA complexes, Science 300 (5625) (2003) 1542-1548.

[21] C. Lucca, et al., Checkpoint-mediated control of replisome-fork association and signalling in response to replication pausing, Oncogene 23 (6) (2004) 1206-1213.

[22] A. Kumagai, et al., TopBP1 activates the ATR-ATRIP complex, Cell 124 (5) (2006) 943-955

[23] S. Matsuoka, et al., ATM and ATR substrate analysis reveals extensive protein networks responsive to DNA damage, Science 316 (5828) (2007) 1160-1166.

[24] A. Marechal, et al., PRP19 transforms into a sensor of RPA-ssDNA after DNA damage and drives ATR activation via a ubiquitin-mediated circuitry, Mol. Cell $53(2)(2014)$ 235-246.

[25] C.S. Wu, L. Zou, The SUMO (Small Ubiquitin-like Modifier) ligase PIAS3 primes ATR for checkpoint activation, J. Biol. Chem. 291 (1) (2016) 279-290.

[26] H.L. Ball, D. Cortez, ATRIP oligomerization is required for ATR-dependent checkpoint signaling, J. Biol. Chem. 280 (36) (2005) 31390-31396.

[27] M. Ambrose, R.A. Gatti, Pathogenesis of ataxia-telangiectasia: the next generation of ATM functions, Blood 121 (20) (2013) 4036-4045.

[28] A.M. Taylor, et al., Ataxia telangiectasia: more variation at clinical and cellular levels, Clin. Genet. 87 (3) (2015) 199-208.

[29] S. Zhang, P. Hemmerich, F. Grosse, Centrosomal localization of DNA damage checkpoint proteins, J. Cell. Biochem. 101 (2) (2007) 451-465.

[30] J. Li, et al., Cytoplasmic ATM in neurons modulates synaptic function, Curr. Biol. 19 (24) (2009) 2091-2096.
[31] L. Valdes-Sanchez, et al., ATR localizes to the photoreceptor connecting cilium and deficiency leads to severe photoreceptor degeneration in mice, Hum. Mol. Genet. 22 (8) (2013) 1507-1515.

[32] A. Roobol, et al., ATR (ataxia telangiectasia mutated- and Rad3-related kinase) is activated by mild hypothermia in mammalian cells and subsequently activates p53, Biochem. J. 435 (2) (2011) 499-508.

[33] H. Ma, T. Pederson, The nucleolus stress response is coupled to an ATR-Chk1-mediated G2 arrest, Mol. Biol. Cell 24 (9) (2013) 1334-1342.

[34] C.A. Lovejoy, D. Cortez, Common mechanisms of PIKK regulation, DNA Repair (Amst.) 8 (9) (2009) 1004-1008.

[35] H. Lempiainen, T.D. Halazonetis, Emerging common themes in regulation of PIKKs and PI3 Ks, EMBO J. 28 (20) (2009) 3067-3073.

[36] T.T. Paull, Mechanisms of ATM activation, Annu. Rev. Biochem 84 (2015) $711-738$.

[37] J. Perry, N. Kleckner, The ATRs, ATMs, and TORs are giant HEAT repeat proteins, Cell 112 (2) (2003) 151-155.

[38] D. Baretic, R.L. Williams, PIKKs-the solenoid nest where partners and kinases meet, Curr. Opin. Struct. Biol. 29 (2014) 134-142.

[39] B.L. Sibanda, D.Y. Chirgadze, T.L. Blundell, Crystal structure of DNA-PKcs reveals a large open-ring cradle comprised of HEAT repeats, Nature 463 (7277) (2010) 118-121.

[40] D.R. Williams, et al., Cryo-EM structure of the DNA-dependent protein kinase catalytic subunit at subnanometer resolution reveals alpha helices and insight into DNA binding, Structure 16 (3) (2008) 468-477.

[41] K. Unsal-Kacmaz, et al., Preferential binding of ATR protein to UV-damaged DNA, Proc. Natl. Acad. Sci. U. S. A. 99 (10)(2002) 6673-6678.

[42] J.W. Luzwick, et al., Mutation of serine 1333 in the ATR HEAT repeats creates a hyperactive kinase, PLoS One 9 (6) (2014) e99397.

[43] A. Grinthal, et al., PR65, the HEAT-repeat scaffold of phosphatase PP2 A, is an elastic connector that links force and catalysis, Proc. Natl. Acad. Sci. U. S. A. 107 (6) (2010) 2467-2472.

[44] Z.H. Wu, S. Miyamoto, Induction of a pro-apoptotic ATM-NF-kappaB pathway and its repression by ATR in response to replication stress, EMBO J. 27 (14) (2008) 1963-1973.

[45] D.A. Mordes, et al., TopBP1 activates ATR through ATRIP and a PIKK regulatory domain, Genes Dev. 22 (11) (2008) 1478-1489.

[46] L.A. Sommer, M. Schaad, S.A. Dames, NMR- and circular dichroism-monitored lipid binding studies suggest a general role for the FATC domain as membrane anchor of phosphatidylinositol 3-kinase-related kinases (PIKK), J. Biol. Chem. 288 (27) (2013) 20046-20063.

[47] X. Jiang, et al., The FATC domains of PIKK proteins are functionally equivalent and participate in the Tip60-dependent activation of DNA-PKcs and ATM, J. Biol. Chem. 281 (23) (2006) 15741-15746.

[48] L.F. DaSilva, et al., The C-terminal residues of Saccharomyces cerevisiae Mec1 are required for its localization: stability, and function, G3 (Bethesda) 3 (10) (2013) 1661-1674.

[49] S. Liu, et al., ATR autophosphorylation as a molecular switch for checkpoint activation, Mol. Cell 43 (2) (2011) 192-202.

[50] E.A. Nam, et al., Thr-1989 phosphorylation is a marker of active ataxia telangiectasia-mutated and Rad3-related (ATR) kinase, J. Biol. Chem. 286 (33) (2011) 28707-28714.

[51] P. Chandris, et al., Compromise in mRNA processing machinery in senescent human fibroblasts: implications for a novel potential role of Phospho-ATR (ser428), Biogerontology 11 (4) (2010) 421-436.

[52] A.J. Prunuske, K.S. Ullman, The nuclear envelope: form and reformation, Curr. Opin. Cell Biol. 18 (1) (2006) 108-116.

[53] M. Zwerger, C.Y. Ho, J. Lammerding, Nuclear mechanics in disease, Annu. Rev. Biomed. Eng. 13 (2011) 397-428.

[54] W.T. Dauer, H.J. Worman, The nuclear envelope as a signaling node in development and disease, Dev. Cell 17 (5) (2009) 626-638.

[55] R. Bermejo, A. Kumar, M. Foiani, Preserving the genome by regulating chromatin association with the nuclear envelope, Trends Cell Biol. 22 (9) (2012) 465-473.

[56] M. Webster, K.L. Witkin, O. Cohen-Fix, Sizing up the nucleus: nuclear shape, size and nuclear-envelope assembly, J. Cell Sci. 122 (Pt. 10) (2009) 1477-1486.

[57] V. Srsen, N. Korfali, E.C. Schirmer, Nuclear envelope influences on cell-cycle progression, Biochem. Soc. Trans. 39 (6) (2011) 1742-1746.

[58] I. Stancheva, E.C. Schirmer, Nuclear envelope: connecting structural genome organization to regulation of gene expression, Adv. Exp. Med. Biol. 773 (2014) 209-244.

[59] J.I. de Las Heras, E.C. Schirmer, The nuclear envelope and cancer: a diagnostic perspective and historical overview, Adv. Exp. Med. Biol. 773 (2014) 5-26.

[60] P. Meinke, E.C. Schirmer, LINC'ing form and function at the nuclear envelope, FEBS Lett. 589 (Pt. A (19)) (2015) 2514-2521.

[61] J. Bozler, et al., Condensins exert force on chromatin-nuclear envelope tethers to mediate nucleoplasmic reticulum formation in Drosophila melanogaster, G3 (Bethesda) 5 (3) (2015) 341-352.

[62] R. Bermejo, et al., The replication checkpoint protects fork stability by releasing transcribed genes from nuclear pores, Cell 146 (2) (2011) 233-246.

[63] X.H. Zhang, C. Zhao, Z.A. Ma, The increase of cell-membranous phosphatidylcholines containing polyunsaturated fatty acid residues induces phosphorylation of p53 through activation of ATR, J. Cell Sci. 120 (Pt. 23) (2007) 4134-4143.

[64] J. Irianto, et al., Osmotic challenge drives rapid and reversible chromatin condensation in chondrocytes, Biophys. J. 104 (4) (2013) 759-769. 
[65] A.M. Martelli, et al., Metabolism and signaling activities of nuclear lipids, Cell. Mol. Life Sci. 61 (10) (2004) 1143-1156.

[66] M. Roshal, et al., Activation of the ATR-mediated DNA damage response by the HIV-1 viral protein R, J. Biol. Chem. 278 (28) (2003) 25879-25886.

[67] C.M. de Noronha, et al., Dynamic disruptions in nuclear envelope architecture and integrity induced by HIV-1 Vpr, Science 294 (5544) (2001) 1105-1108.

[68] G. Jacquot, et al., Localization of HIV-1 Vpr to the nuclear envelope: impact on Vpr functions and virus replication in macrophages, Retrovirology 4 (2007) 84.

[69] V. Planelles, S. Benichou, Vpr and its interactions with cellular proteins, Curr. Top. Microbiol. Immunol. 339 (2009) 177-200.

[70] S. Boulon, et al., The nucleolus under stress, Mol. Cell 40 (2) (2010) 216-227.

[71] D.H. Larsen, M. Stucki, Nucleolar responses to DNA double-strand breaks, Nucleic Acids Res. 44 (2) (2016) 538-544.

[72] S. Sengupta, T.R. Peterson, D.M. Sabatini, Regulation of the mTOR complex 1 pathway by nutrients: growth factors, and stress, Mol. Cell 40 (2) (2010) 310-322.

[74] M. Sokka, et al., High levels of TopBP1 induce ATR-dependent shut-down of rRNA transcription and nucleolar segregation, Nucleic Acids Res. 43 (10) (2015) 4975-4989.

[75] Perez-Castro, A.J.R. Freire, Rad9 B responds to nucleolar stress through ATR and JNK signalling, and delays the G1-S transition, J. Cell Sci. 125 (Pt. 5) (2012) 1152-1164.

[76] C.J. Sherr, et al., p53-Dependent and -independent functions of the Arf tumor suppressor, Cold Spring Harb. Symp. Quant. Biol. 70 (2005) 129-137.

[77] S. Rocha, K.J. Campbell, N.D. Perkins, p53- and Mdm2-independent repression of NF-kappa B transactivation by the ARF tumor suppressor, Mol. Cell 12 (1) (2003) 15-25.

[78] M.L. Kuo, et al., Arf induces p53-dependent and -independent antiproliferative genes, Cancer Res. 63 (5) (2003) 1046-1053.

[79] S. Rocha, et al., Regulation of NF-kappaB and p53 through activation of ATR and Chk1 by the ARF tumour suppressor, EMBO J. 24 (6) (2005) 1157-1169.

[80] F. Wei, et al., ERK1 and ERK2 kinases activate hydroxyurea-induced S-phase checkpoint in MCF7 cells by mediating ATR activation, Cell. Signal. 23 (1) (2011) 259-268.

[81] D. Wu, et al., ERK activity facilitates activation of the S-phase DNA damage checkpoint by modulating ATR function, Oncogene 25 (8) (2006) 1153-1164

[82] E.A. Nigg. J.W. Raff, Centrioles, centrosomes, and cilia in health and disease, Cell 139 (4) (2009) 663-678.

[83] P.T. Conduit, A. Wainman, J.W. Raff, Centrosome function and assembly in animal cells, Nat. Rev. Mol. Cell Biol. 16 (10) (2015) 611-624.

[84] M.K.A. Shimada, Kobayashi The Potential Roles of DNA-Repair Proteins in Centrosome Maintenance, InTech, 2011.

[85] M. Shimada, et al., Inactivation of the Nijmegen breakage syndrome gene leads to excess centrosome duplication via the ATR/BRCA1 pathway, Cancer Res. 69 (5) (2009) 1768-1775

[86] E. Griffith, et al., Mutations in pericentrin cause Seckel syndrome with defective ATR-dependent DNA damage signaling, Nat. Genet. 40 (2) (2008) $232-236$
[87] A. Tibelius, et al., Microcephalin and pericentrin regulate mitotic entry via centrosome-associated Chk1, J. Cell Biol. 185 (7) (2009) 1149-1157.

[88] A. Rauch, et al., Mutations in the pericentrin (PCNT) gene cause primordial dwarfism, Science 319 (5864) (2008) 816-819.

[89] A. Klingseisen, A.P. Jackson, Mechanisms and pathways of growth failure in primordial dwarfism, Genes Dev. 25 (19) (2011) 2011-2024.

[90] M. Brauchle, K. Baumer, P. Gonczy, Differential activation of the DNA replication checkpoint contributes to asynchrony of cell division in $C$. elegans embryos, Curr. Biol. 13 (10) (2003) 819-827.

[91] L. Smith, et al., Duplication of ATR inhibits MyoD: induces aneuploidy and eliminates radiation-induced G1 arrest, Nat. Genet. 19 (1) (1998) 39-46.

[92] C. Mori, T. Takanami, A. Higashitani, Maintenance of mitochondrial DNA by the Caenorhabditis elegans ATR checkpoint protein ATL-1, Genetics 180 (1) (2008) 681-686.

[93] K. Suetomi, et al., Caenorhabditis elegans ATR checkpoint kinase ATL-1 influences life span through mitochondrial maintenance, Mitochondrion 13 (6) (2013) 729-735.

[94] H.S. Tivey, et al., Small molecule inhibition of p38 MAP kinase extends the replicative life span of human ATR-Seckel syndrome fibroblasts, J. Gerontol. A. Biol. Sci. Med. Sci. 68 (9) (2013) 1001-1009.

[95] A.A. Kiger, et al., A functional genomic analysis of cell morphology using RNA interference, J. Biol. 2 (4) (2003) 27.

[96] R.D. Beveridge, et al., The leukemia-associated Rho guanine nucleotide exchange factor LARG is required for efficient replication stress signaling, Cell Cycle 13 (21) (2014) 3450-3459.

[97] E. Ericson, et al., A novel role of the checkpoint kinase ATR in leptin signaling, Mol. Cell. Endocrinol. 412 (2015) 257-264.

[98] H. Mokrani-Benhelli, et al., Primary microcephaly: impaired DNA replication, and genomic instability caused by compound heterozygous ATR mutations, Hum. Mutat. 34 (2) (2013) 374-384.

[99] M.H. de Ru, et al., Interstitial deletion in 3q in a patient with blepharophimosis-ptosis-epicanthus inversus syndrome (BPES) and microcephaly, mild mental retardation and growth delay: clinical report and review of the literature, Am. J. Med. Genet. A 137 (1) (2005) 81-87.

[100] M. Murga, et al., A mouse model of ATR-Seckel shows embryonic replicative stress and accelerated aging, Nat. Genet. 41 (8) (2009) 891-898.

[101] Y. Ruzankina, et al., Deletion of the developmentally essential gene ATR in adult mice leads to age-related phenotypes and stem cell loss, Cell Stem Cell 1 (1) (2007) 113-126.

[102] L.B. Gordon, et al., Progeria: a paradigm for translational medicine, Cell 156 (3) (2014) 400-407.

[103] M. Murga, et al., Exploiting oncogene-induced replicative stress for the selective killing of Myc-driven tumors, Nat. Struct. Mol. Biol. 18 (12) (2011) $1331-1335$.

[104] E. Fokas, et al., Targeting ATR in DNA damage response and cancer therapeutics, Cancer Treat Rev. 40 (1) (2014) 109-117.

[105] A. Tanaka, et al., Germline mutation in ATR in autosomal- dominant oropharyngeal cancer syndrome, Am. J. Hum. Genet. 90 (3) (2012) 511-517. 